\title{
Error threshold in RNA quasispecies models with complementation
}

\author{
Josep Sardanyés ${ }^{1,2, *}$ and Santiago F. Elena ${ }^{1,3}$ \\ ${ }^{1}$ Instituto de Biología Molecular y Celular de Plantas, \\ Consejo Superior de Investigaciones Científicas-UPV, \\ Ingeniero Fausto Elio s/n, 46022 València, Spain \\ ${ }^{2}$ Complex Systems Lab (ICREA-UPF), Barcelona Biomedical Research Park (PRBB), Dr. Aiguader 88, 08003 Barcelona, Spain \\ ${ }^{3}$ Santa Fe Institute, 1399 Hyde Park Road, Santa Fe NM 87501, USA
}

(Dated: April 26, 2010)

\begin{abstract}
A general assumption of quasispecies models of replicons dynamics is that the fitness of a genotype is entirely determined by its sequence. However, a more biologically plausible situation is that fitness depends on the proteins that catalyze metabolic reactions, including replication. In a stirred population of replicons, such as viruses replicating and accumulating within the same cell, the association between a given genome and the proteins it encodes is not tight as it can be replicated by proteins translated from other genomes. We have investigated how this complementation phenomenon affects the error threshold in simple quasispecies mean field models. We first studied a model in which the master and the mutant genomes code for wild-type and mutant replicases, respectively. We assume that the mutant replicase has a reduced activity and that that the wild-type replicase does not have increased affinity for the master genome. The whole pool of replicases can bind and replicate both genomes. We then analyze a different model considering a more extreme case of mutant genomes, the defective interfering particles (DIPs) described in many cases of viral infection. DIPs, with a higher replication rate owed to their shorter genomes, do not code for replicase, but they are able of using the replicase translated from the master genome. Our models allow to study how the probability of interaction between the genomes and the whole pool of replicases affects the error threshold. In both systems we characterize the scenario of coexistence between master and mutant genomes, providing the critical values of mutation rate, $\mu_{c}$, and the critical interaction rate between master genomes and replicases, $\gamma_{c}$, at which the quasispecies enters into error catastrophe, a situation in which the mutant genomes dominate the population. In both cases, we showed that the error-threshold transition is given by transcritical-like bifurcations, suggesting a continuous phase transition. We have also found that the region in the parameter space $(\mu, \gamma)$ in which the master sequence survives is reduced when DIPs are introduced into the system.
\end{abstract}

Keywords: complementation, defective interfering particles, error threshold, quasispecies theory, RNA viruses, systems biology

\section{INTRODUCTION}

RNA viruses are the most important pathogens infecting plants and animals, and they are also a continuous source of emerging infectious diseases. The extremely short generation time, large population size and high mutation rates make RNA viruses excellent tools for experimental evolution and for testing basic principles of Evolutionary Theory [14]. RNA viruses are characterized as highly polymorphic populations which are usually assimilated to quasispecies by many virologists $[1-3]$. The quasispecies population structure arises as a consequence of fast replication coupled with high mutation rates $[4-7]$. The mathematical quasispecies theory [811] describes populations of replicons as a collection of a master genomes and a diverse cloud of mutants, which all together contribute to the phenotypic properties of

*Author for correspondence: Instituto de Biología Molecular y Celular de Plantas, Consejo Superior de Investigaciones CientíficasUPV, Ingeniero Fausto Elio s/n, 46022 València, Spain. E-mail: josep.sardanes@upf.edu; Phone: 34963878 638; Fax: 34963877 859 the entire population. Under this view, the entire quasispecies, rather than individuals, is the target of natural selection $[12,13]$. A remarkable result of the quasispecies theory is the prediction of the existence of a phase transition at a critical mutation rate, $\mu_{c}$, the error-threshold, beyond which the master sequence is lost and the quasispecies is dominated by the mutant genomes. Roughly speaking, the critical mutation rate can be obtained as $\mu_{c} \approx \nu^{-1}$, being $\nu$ the sequence length. The theory predicts that replicons with mutation rates $\mu>\mu_{c}$, might enter into the so-called error catastrophe regime, involving the out-competition of the master sequence by the pool of mutant genomes. Hence, genomic information is lost as the population enters into a drift phase $[8,15]$.

The standard quasispecies mathematical model assumes that the fitness of any particular genotype entirely depends on its genome, without specifically distinguishing between effects at the genotypic and protein (phenotypic) levels. However, this assumption is highly unrealistic, because the fitness of a genome would depend on the functionality of the proteins it encodes. Furthermore, as master and mutant genomes coexist and both may contribute to the pool of proteins, it is conceivable that wild-type proteins may, for instance, act over mutant genomes, replicating and/or encapsidating them and 

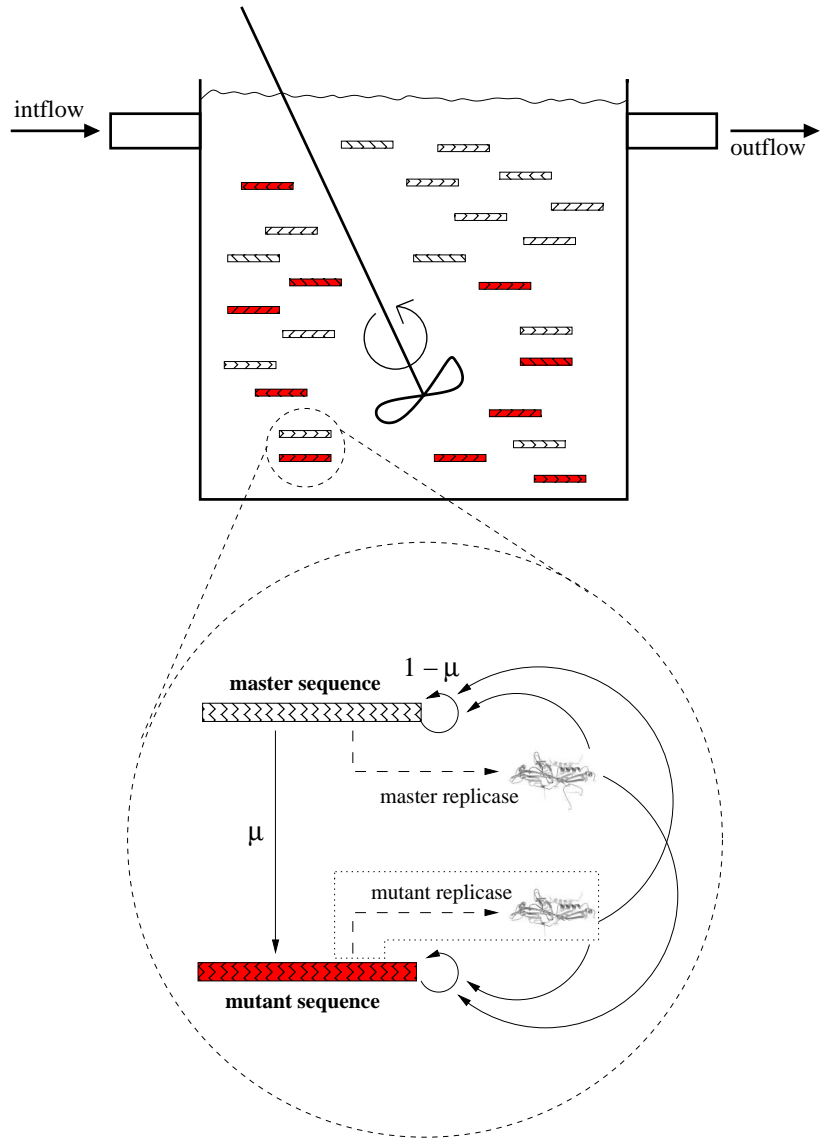

FIG. 1: Schematic representation of the flow reactor approach used as abstract model to study quasispecies dynamics with complementation. The master (white) and the mutant (red) genomes encode for wild-type and mutant replicases, respectively. In Section II we consider the case where the mutant genomes code for deficient replicase with a reduced polymerization activity. In Section III we study the dynamics considering the mutant genomes as defective interfering particles (DIPs), which do not encode for a replicase (i.e., the process framed with the dotted line is removed). In both cases, the mutant genomes take advantage of the wild-type replicase to make more copies of themselves. Both systems are equivalent to a two-member hypercycle formed by a replicator and a parasite, which share the replicase (solid and dashed arrows indicate, respectively, replication and translation processes).

vice versa, in a clear case of functional trans complementation [16]. Henceforth, the phenotype of a genome may not reflect its genotype, a situation which in the case of viruses is known as phenotypic mixing and hiding [17-20]. Moreover, a substantial amount of evidences, gathered with viruses as different as the murine cytomegalovirus [21], foot-and-mouth disease virus [22] or tobacco mosaic virus [23], give support to the fact that deletion mutants can be replicated in cells coinfected with the full-genome helper viruses.

Several mathematical models have been proposed that take into consideration trans interactions between different viral genomes with different fitness properties [24].
For instance, studying the dynamics of defective interfering particles (DIPs) as an extreme case of complementation [25-29]. DIPs are mutant viruses that lack most of the viral genome and cannot complete the infectious cycle by themselves [30, 31] (see also [32] for a review). However, they can be replicated and encapsidated by the proteins translated from a helper virus coinfecting the same cell. The mechanisms ensuring the survival and persistence of DIPs are not entirely clear, as they behave as hyperparasites and usually get involve in an arms race with the full virus [33-35]. It is possible that the emergence of genomes shortened by deletions might confer an advantage in terms of replication speed compared with the full virus [22]. Furthermore, there is also evidence of a stronger form of interference whereby the DIPs genome competes more successfully for the viral replicative machinery $[34,36]$.

As previously mentioned, several authors have theoretically investigated the dynamics of full viruses replicating together with DIPs. For example, Szathmáry [28] analyzed simple models based on mass action kinetics considering standard viruses and DIPs. In [28], structured deme models where developed to provide a description of the coexistence of virus segments considering standard virus and DIPs, sensitive and resistant viruses together with DIPs, covirus pairs (i.e., virus that exist as two or more separated particles all of which must be present for the complete replication cycle of a virus to occur), and virus-covirus systems. A deeper analysis of the model with standard virus and DIPs was later developed by Szathmáry [29] considering cell populations infected by particles differing in number. Several different coexistence situations were shown to be possible by means of stable equilibria governed by fixed points or periodic orbits; also evidence was found of stable coexistence out of equilibrium governed by strange attractors.

Later, Kirkwood et al [27] developed a differential equations model to analyze a system formed by host cells, wild-type virions and DIPs. Such a model was able to explain several dynamic behaviors found in experiments, especially the fluctuations in virus titers on successive passages. Their model also gathered some interesting dynamical phenomena such as self-curing, which involves the extinction of the full virus together with the DIPs, and that had been previously observed to occur in vitro [37]. They found that self-curing was associated to transient chaos, which might arise due to the presence of chaotic saddles in phase space.

More recently, Wilke et al [16], analyzed a simple model of complementation with differential fitness between master and mutant viruses due to an impaired ability to infect cells. The model studied by these authors predicted a strong influence of phenotypic mixing and hiding on population dynamics of viruses at high multiplicity of infection as well as important effects on the mutation-selection balance at low multiplicity of infection.

None of the aforementioned models, and to the ex- 
(a)

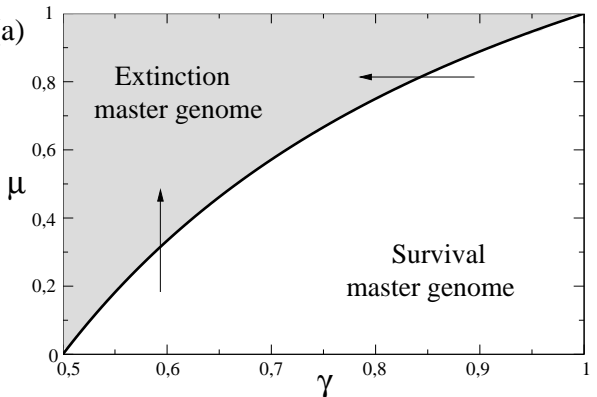

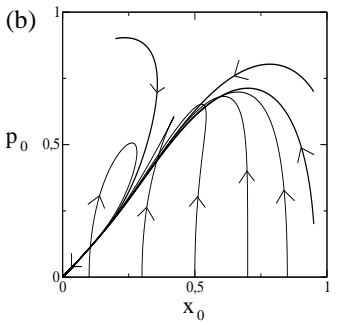
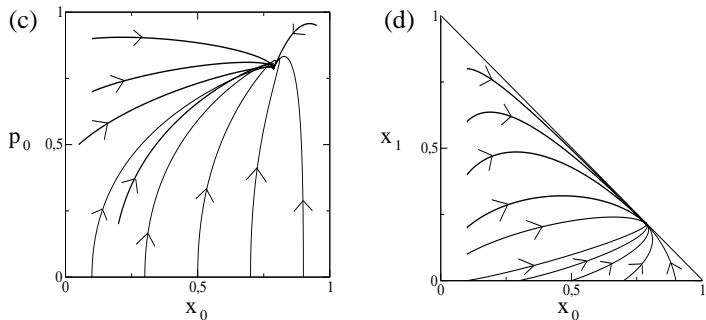

FIG. 2: Dynamics generated by the model given by Eqs. (1-3). (a) Phase diagram showing the region in the parameter space $(\gamma, \mu)$ in which the master sequence extincts (gray region, with parameters $k_{0}=k_{1}=0.4$ ). The solid line indicates the errorthreshold boundary, which can be achieved by increasing $\mu$ or decreasing $\gamma$. This critical curve perfectly fits with the analytical values of $\mu_{c}=2-\gamma^{-1}$ and $\gamma_{c}=(2-\mu)^{-1}$, obtained from linear stability analysis. We also display some phase portraits showing several trajectories (the arrows show the directions of the flows) on the phase plane $\left(x_{0}, p_{0}\right)$ with: (b) $k_{0}=k_{1}=0.4$ and $\gamma=1 / 2$; (c) $k_{0}=k_{1}=0.4$ and $\gamma=0.75$. In (d) we show the dynamics of the master and the mutant genomes in the simplex $\left(x_{0}, x_{1}\right)$, here with parameters $k_{0}=0.8, k_{1}=0.4$ and $\gamma=0.75$. In all the plots we used $r_{0}=0.9, r_{1}=0.1$ and $\mu=0.3$. Thin trajectories correspond to the biologically meaningful initial conditions with $0<x_{0}(0) \leq 1$ and $x_{1}(0)=p_{0}(0)=p_{1}(0)=0$. In (b) and (c) we used $x_{1}(0)=p_{1}(0)=0$; in $(\mathrm{d})$ we used $p_{0}(0)=p_{1}(0)=0$. All the numerical results are obtained with the fourth-order Runge-Kutta method with a constant time step-size $\delta t=0.1$.

tend of our knowledge no one else, explores the effect of complementation on the error threshold predicted by the standard quasispecies theory. Therefore, neither the parametric regions in which the master sequence survives nor the bifurcations causing the error threshold have been characterized for systems of replicons in which complementation between mutant and master genomes may occur. We sought to cover this hole by studying two simple quasispecies models taking into account complementation during replication between master and mutant genomes.

The model studied in Section II considers that both master and mutant genomes encode, respectively, for wild-type and mutant replicases, with the mutant replicase having a lower catalytic activity. Hence, the fitness landscape is included at the replicase level and is given by the Swetina-Schuster fitness landscape [42]. However, since both types of replicases contribute to the cellular pool of proteins, both can replicate the master and mutant genomes. From a more biologically relevant perspective, the model can also give insights on the population dynamics of master and mutant viruses replicating within the same cell and may help to understand under which conditions master genomes can survive in the population.

The second model, developed in Section III, considers a problem of relevance in virology: the production of DIPs by some viruses and the effect they may exert on the full-length virus. In this model DIPs do not encode for replicase and have a faster replication rate and/or higher effective interaction with the wild-type replicase. Such a model will also provide some insights into the effect of competition between master genomes and DIPs, as well as into the possible persistence scenarios of the master sequence under the presence of DIPs. In both systems, replication is governed by a nonlinear, density-dependent growth. Actually, this system is equivalent to a two- member hypercycle with replicase-mediated replication (see Fig. 1). Some models of hypercycles coding for a replicase able to instruct the synthesis of other replicators can be found in [38].

\section{QUASISPECIES MODEL WITH COMPLEMENTATION}

We analyze the effect of complementation in the dynamics of RNA viruses with a simple quasispecies mean field model that describes the replication-mutation dynamics of RNA macromolecules in a flow reactor (see Fig. 1). Hence we study an unstructured model that only gathers the replication kinetics with a constant population, obviating the details of viral intracellular amplification. This is a standard method used in replicator theoretical models (see e.g., [39-41]). For mathematical convenience we studied the Swetina-Schuster single peak fitness landscape [42], where mutations are assumed to be largely deleterious and all mutant genotypes have identical low fitness. For simplicity we will not consider beneficial or neutral mutations. Our model considers the simplest scenario dividing the population of genomes in two types of sequences, the master and the mutant genomes, which are grouped into an "average" mutant sequence. The relative concentrations for such sequences are denoted $x_{0} \geq 0$ (master) and $x_{1} \geq 0$ (mutant), respectively. Such sequences are assumed to be positivesense strands that encode, respectively, for the wild-type (denoted as $p_{0} \geq 0$ ) and the mutant replicases (named $\left.p_{1} \geq 0\right)$. Hence, the master genomes are considered to be the sequences coding for the more efficient replicases. As a first approach we obviated the polarity of both sequences (i.e., genomic and antigenomic strands) not considering the effect of the intermediaries of replication. 
The model assumes a constant population $(\mathrm{CP})$ constraint with $\sum_{i=0}^{1} x_{i}=1$ and $\sum_{i=0}^{1} p_{i}=1$. Let us also assume that mutations occur from the master to the second compartment but not in the reverse sense. The enormous size of the sequence space makes this assumption a first good approximation. The model is then given by the following set of differential equations:

$$
\begin{gathered}
\dot{x}_{0}=(1-\mu) x_{0} \gamma\left[r_{0} p_{0}+r_{1} p_{1}\right]-x_{0} \Phi(\mathbf{x}), \\
\dot{x}_{1}=\left(\mu \gamma x_{0}+(1-\gamma) x_{1}\right)\left[r_{0} p_{0}+r_{1} p_{1}\right]-x_{1} \Phi(\mathbf{x}), \\
\dot{p}_{i}=k_{i} x_{i}-p_{i} \Xi(\mathbf{p}),
\end{gathered}
$$

with $i=0,1$. The outflux terms, which are computed from $\sum_{i=0}^{1} \dot{x}_{i}=0$ and $\sum_{i=0}^{1} \dot{p}_{i}=0$, are given by

$$
\Phi(\mathbf{x})=\left(x_{0} \gamma+(1-\gamma) x_{1}\right)\left[r_{0} p_{0}+r_{1} p_{1}\right],
$$

and $\Xi(\mathbf{p})=\sum_{i=0}^{1} k_{i} x_{i}$. Note that we use two different outfluxes because the RNA genomes and the replicase enzymes are competing for different resources (i.e., mononucleotides and amino acids, respectively). Here $r_{0}>0$ and $r_{1}>0$ are the replication rates due to the action of the wild-type and mutant replicases, respectively. We will consider that the mutant genome will always produce a mutant replicase whith a replication rate $r_{1} \leq r_{0}$. Note that the term $\left(r_{0} p_{0}+r_{1} p_{1}\right)$ appears symmetrically in Eqs. (1)-(2) because of complementation. Hence, both replicases, the master and the mutant, are able to bind and replicate both genome classes. The parameter $\mu$, associated to the replication kinetics, is the average mutation rate. Finally, the parameter $k_{i}$ denote the translation rate of the replicases from both master and mutant genomes.

Finally, the parameter $\gamma>0$ is the probability of interaction between the pool of replicases (i.e., wild-type and mutant replicases) and the master genomes, being $(1-\gamma)$ the interaction probability between the mutant genomes and the pool of replicases. Our model allows to consider the effect of mutations at the level of the replicase and of the genomes, depending on the value of $\gamma$ : with $\gamma=0.5$ the replicase will interact with the same probability with the master and the mutant genomes. For this case, the deleterious effects of mutations are only incorporated at the level of the replicase. However, with $\gamma>0.5$ the pool of replicases will undergo a better interaction with the master genomes, and the effect of mutations on the fitness will be also reflected at the level of the genotype. For the moment, we will not consider the case where the mutant genomes have more affinity for the pool of replicases (i.e., $\gamma<0.5)$.

The CP constraint allows reducing the previous model to a two-dimensional system because of the linear relations $x_{1}=1-x_{0}$ and $p_{1}=1-p_{0}$. Hence, we can
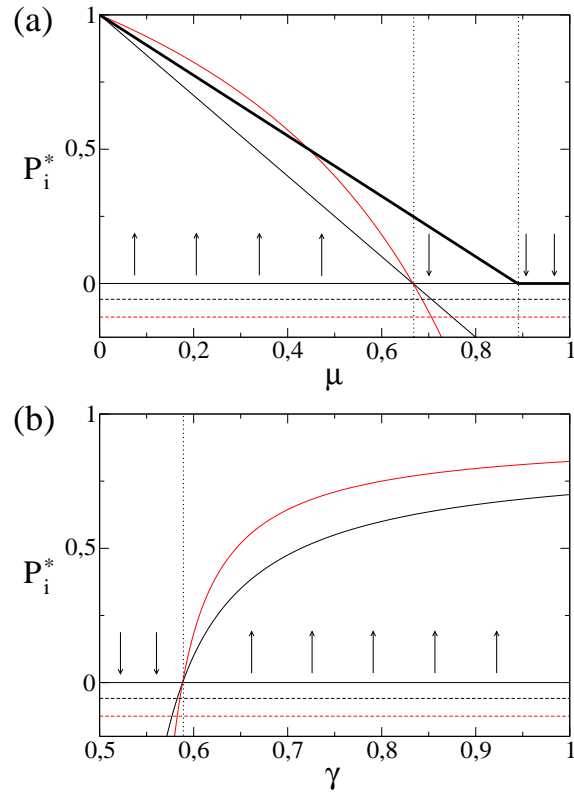

FIG. 3: Values of the coordinates of the fixed points, $P_{i}^{*}$ $(i=1 \ldots 3)$, for the two-dimensional model at increasing $\mu$ (a) and $\gamma(\mathrm{b})$ with $k_{0}=0.8, k_{1}=0.4, r_{0}=0.9$ and $r_{1}=0.1$ (in black we show the coordinate $x_{0}^{*}$ and in red the coordinate $p_{0}^{*}$ ). We indicate $P_{2}^{*}$ and $P_{3}^{*}$ with solid and dashed lines, respectively. Note that $P_{3}^{*}$ remains negative in both panels. The intersections of the two lines (i.e., black and red) between fixed points are bifurcations. In (a) we use $\gamma=0.75$ and in (b) we use $\mu=0.3$. The arrows indicate the stability of the extinction equilibrium $P_{1}^{*}$, which is indicated with a solid thin line. For comparison, we show the prediction of the error threshold for a simple quasispecies model without complementation, computing numerically the concentration of the master genomes (solid thick line) from Eqs. (6)-(7) using $f_{0}=0.9, f_{1}=0.1$ and $x_{0}(0)=1$ and $x_{1}(0)=0$ as initial conditions. The critical values (e.g., $\mu_{c}$ ) involving the transition from viable to non-viable master genomes are indicated with a vertical dotted line.

study the time dynamics of the master genomes and the wild-type replicase, now defined in the phase plane $\Gamma:=\left\{\left(x_{0}, p_{0}\right) \in \mathbb{R}^{2}: 0 \leq x_{0}, p_{0} \leq 1\right\}$. The reduced model is given by

$$
\begin{gathered}
\dot{x}_{0}=(1-\mu) x_{0} \gamma\left[r_{0} p_{0}+r_{1}\left(1-p_{0}\right)\right]-\Phi(\mathbf{x}) x_{0}, \\
\dot{p}_{0}=k_{0} x_{0}-p_{0}\left(k_{0} x_{0}+k_{1}\left(1-x_{0}\right)\right) .
\end{gathered}
$$

Now with $\Phi(\mathbf{x})=\left(x_{0} \gamma+(1-\gamma)\left(1-x_{0}\right)\right)\left[r_{0} p_{0}+\right.$ $\left.r_{1}\left(1-p_{0}\right)\right]$. This two-dimensional model has three equilibrium points. The first fixed point is the trivial one, $P_{1}^{*}=(0,0)$, which involves the extinction of the master genomes. Such a case would correspond to an absorbing state, in which the system remains trapped forever. The other two equilibria are $P_{2}^{*}=\left(x_{0}^{*(2)}=1+\gamma \mu /(1-\right.$ $\left.2 \gamma), p_{0}^{*(2)}=k_{0}(1+\gamma(\mu-2)) /\left(k_{0}(1+\gamma(\mu-2))-\gamma k_{1} \mu\right)\right)$ and 
(a)

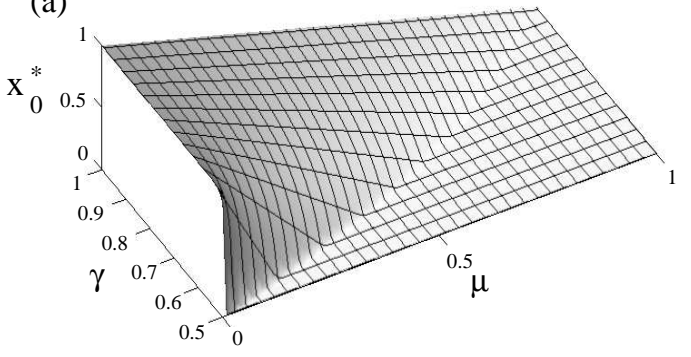

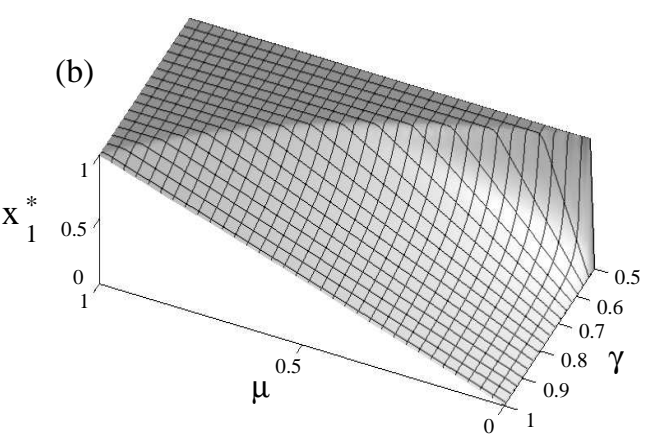

FIG. 4: Equilibrium concentration of the master (a) and mutant (b) genomes numerically computed from the four-dimensional system (1-3) in the parameter space $(\mu, \gamma)$, using $r_{0}=1, r_{1}=0.1, k_{0}=0.8$ and $k_{1}=0.4$. Initial conditions were: $x_{0}(0)=1$ and $x_{1}(0)=p_{0}(0)=p_{1}(0)=0$.

$P_{3}^{*}=\left(x_{0}^{*(3)}=k_{1} r_{1} /\left(k_{1} r_{1}-k_{0} r_{0}\right), p_{0}^{*(3)}=r_{1} /\left(r_{1}-r_{0}\right)\right)$. We note that under the $\mathrm{CP}$ constraint, the fixed points for Eqs. (1-3) are fully determined and are given by: $P_{1}^{*}=(0,1,0,1), P_{2}^{*}=\left(x_{0}^{*(2)}, 1-x_{0}^{*(2)}, p_{0}^{*(2)}, 1-p_{0}^{*(2)}\right)$ and $P_{3}^{*}=\left(x_{0}^{*(3)}, 1-x_{0}^{*(3)}, p_{0}^{*(3)}, 1-p_{0}^{*(3)}\right)$. According to the equilibrium values for the two-dimensional system, when $k_{1}=k_{0}$ the fixed points $P_{2}^{*}$ and $P_{3}^{*}$ live on the lines $x_{0}^{*(2)}=p_{0}^{*(2)}$ and $x_{0}^{*(3)}=p_{0}^{*(3)}$, respectively. The Jacobian matrix for Eqs. (4-5) is given by

$$
J=\left(\begin{array}{cc}
\partial \dot{x}_{0} / \partial x_{0} & \partial \dot{x}_{0} / \partial p_{0} \\
p_{0}\left(k_{1}-k_{0}\right)+k_{0} & x_{0}\left(k_{1}-k_{0}\right)-k_{1}
\end{array}\right)
$$

with $\partial \dot{x}_{0} / \partial x_{0}=-\left(p_{0}\left(r_{0}-r_{1}\right)+r_{1}\right)\left(1-2 x_{0}+\gamma(-2+\mu+\right.$ $\left.\left.4 x_{0}\right)\right)$ and $\partial \dot{x}_{0} / \partial p_{0}=-\left(r_{0}-r_{1}\right) x_{0}\left(1-x_{0}+\gamma(-2+\mu+\right.$ $\left.\left.2 x_{0}\right)\right)$. In order to analytically find the critical mutation rate, $\mu_{c}$, responsible of the error-threshold, we study the stability of the trivial fixed point $P_{1}^{*}$, which, if stable, will involve the extinction of the master sequence. The Jacobian matrix evaluated at the trivial equilibrium takes the form

$$
J(\mathbf{0})=\left(\begin{array}{cc}
r_{1}((1-\mu) \gamma+(\gamma-1)) & 0 \\
k_{0} & -k_{1}
\end{array}\right) .
$$

The stability is studied from $\operatorname{det}\left|J\left(P_{1}^{*}\right)-\lambda \mathrm{I}\right|=0$. We obtain two eigenvalues: $\lambda^{(1)}=(1-\mu) \gamma r_{1}+r_{1}(\gamma-1)$ and $\lambda^{(2)}=-k_{1}$. Note that $\lambda^{(2)}$ is always negative, and thus the stability of this fixed point will entirely depend on $\lambda^{(1)}$. The trivial fixed point, which involves the extinction of the master genomes, will be stable when $\mu>\mu_{c}$ or when $\gamma<\gamma_{c}$, with $\mu_{c}=2-\gamma^{-1}$ and $\gamma_{c}=(2-\mu)^{-1}$, respectively. When $\gamma=1 / 2$ the critical mutation rate is $\mu_{c}=0$, and then, for any value of the mutation rate $\mu>0$, the master sequence is not able to survive. In Fig. 2 we display the dynamics obtained numerically from the full model with four variables. The critical boundary involving the error threshold and the extinction of the master genomes is shown in the phase diagram of Fig. 2 (a). Here we computed the pairs of $\mu$ and $\gamma$ where the master genome is able to persist for $t \leq 10^{5}$ (white region). The solid line indicates the critical values of each of these parameters causing the error threshold phase transition. This curve perfectly fits with the analytical expressions of $\mu_{c}$ and $\gamma_{c}$ previously derived from linear stability analysis. For this model, values of $\gamma<1 / 2$ involve the extinction of the master genome independently of $\mu$ (results not shown). The dynamics with $\gamma=1 / 2$ is illustrated in Fig. 2(b) by means of two-dimensional phase. For this case the master sequence extincts, being the entire phase plane $\left(x_{0}, p_{0}\right)$ a basin of attraction of the point $(0,0)$, which is globally stable. The exploration of the phase portraits using $\gamma>1 / 2$ shows the non-trivial equilibrium of the master genome, and thus the coexistence of the master and the mutant genomes (see Figs. $2(\mathrm{c})$ and $2(\mathrm{~d}))$.

If we now focus again in the reduced, two-dimensional model we can compute the values of the coordinates of the fixed points $P_{2,3}^{*}$ at increasing $\mu$ and $\gamma>1 / 2$. In Fig. 3(a) we show the values of such coordinates using $\mu$ as control parameter. We saw a linear decrease of the equilibrium concentration for the master genome, corresponding to the first coordinate of the fixed point $P_{2}^{*}$. This decrease was followed by the coordinate $p_{0}^{*(2)}$, because the production of wild-type replicase is proportional to the concentration of master genomes. The two coordinates of the equilibrium $P_{3}^{*}$ remain negative for the parameter values used in Fig. 3. Similar results are shown in Fig. 3(b) using $\gamma$ as control parameter. As $\gamma$ decreases, the equilibria for the master genome and for the wild-type replicase decreased, and after the bifurcation value $\gamma=\gamma_{c}$ (with $\gamma<\gamma_{c}$ ) the master genomes are not able to persist.

The results shown in Figs. 3(a) and 3(b) display a transcritical bifurcation which may govern a continuous phase transition involved in the error threshold. This bifurcation can be inferred from the intersections of the lines corresponding to the fixed points $P_{1}^{*}$ and $P_{2}^{*}$ and 

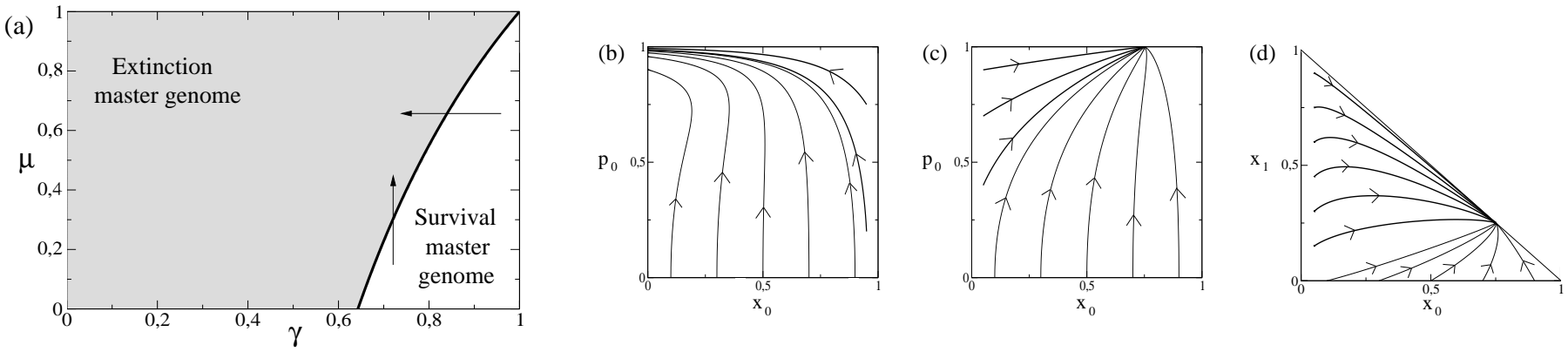

FIG. 5: Same as in Fig. 2 but now considering the population dynamics with mutant genomes being DIPs and using Eqs. (10)(12). (a) Phase diagram with the critical boundary in the parameter space $(\gamma, \mu)$ involved in the error threshold (black line). This boundary perfectly matches with the critical parameter values analytically derived, and given by $\mu_{c}=1-\left((1-\gamma) r_{1} / \gamma r_{0}\right)$ and $\gamma_{c}=r_{1} /\left(r_{0}(1-\mu)+r_{1}\right)$. The phase portraits are displayed for: (b) $\mu=0.4$ and $\gamma=0.6$; (c) and (d) $\mu=0.2$ and $\gamma=0.9$. In all the plots we use $r_{0}=0.5, r_{1}=0.9$ and $k_{0}=0.5$. In (b) and (c) $x_{1}(0)=0$ and in (d) $p_{0}(0)=0$.

from the stability properties of such points. These intersections indicate that these two fixed points collide at the critical values $\mu_{c}=2 / 3$ (a) and $\gamma_{c} \approx 0.588$ (b), which are in agreement with the critical values of these parameters analytically derived. Actually, we generically obtain

$$
\left.P_{2}^{*}\right|_{\mu_{c}=2-\frac{1}{\gamma}}=(0,0) \quad \text { and }\left.\quad \mathrm{P}_{2}^{*}\right|_{\gamma_{\mathrm{c}}=(2-\mu)^{-1}}=(0,0) \text {. }
$$

Hence, $P_{2}^{*}=P_{1}^{*}$ for the critical values of $\mu$ and $\gamma$, indicating that such points collide and the bifurcation occurs. After the collision, the coordinate $x_{0}^{*}$ of the fixed point $P_{2}^{*}$ is negative, being out of $\Gamma$. Numerical computations show that after the collision, these two fixed points interchange the stability properties: with $\mu<\mu_{c}$ the fixed point $P_{1}^{*}$ is unstable, while for $\mu>\mu_{c}$ becomes stable, as previously shown by the linear stability analysis. The stability of $P_{2}^{*}$ works in an opposite way, that is, when $\mu<\mu_{c}, P_{2}^{*}$ is stable, and with $\mu>\mu_{c}$ is unstable.

To better illustrate the effect of complementation in the behavior of the error threshold transition, we constructed the standard quasispecies model under the CP constraint (i.e., $x_{0}+x_{1}=1$ ) and the Swetina-Schuster fitness landscape. The dynamical system, following similar notation than for the previous model, is given by

$$
\dot{x}_{0}=f_{0}(1-\mu) x_{0}-x_{0} \eta(\mathbf{x}),
$$

$$
\dot{x}_{1}=f_{0} \mu x_{0}+f_{1} x_{1}-x_{1} \eta(\mathbf{x}) .
$$

now with the outflow term given by $\eta(\mathbf{x})=\sum f_{i} x_{i}$. Here $f_{0}$ and $f_{1}$ are the replication rates of the master $\left(x_{0}\right)$ and the mutant $\left(x_{1}\right)$ genomes, respectively. Equations (6)-(7) can be reduced to a one-dimensional system, given by

$$
\dot{x}_{0}=f_{0} x_{0}\left(1-\mu-\frac{f_{1}}{f_{0}}-x_{0}\left(1-\frac{f_{1}}{f_{0}}\right)\right) .
$$

Actually, we can compute the time evolution of this single equation, which gives a logistic-like solution

$$
x_{0}(t)=\frac{\omega_{0}}{\omega_{1}}\left[1+\left(\frac{\omega_{0} / \omega_{1}-x_{0}(0)}{x_{0}(0)}\right) \exp \left(-\omega_{0} f_{0} t\right)\right]^{-1},
$$

with $\omega_{0}=1-\mu-f_{1} / f_{0}$ and $\omega_{1}=1-f_{1} / f_{0}$. The critical mutation rate for this simple quasispecies model without complemention, involving the extinction of the master genome is given by

$$
\mu_{c}=1-f_{1} / f_{0}
$$

To compare the critical mutation rate with and without complementation we compute the equilibrium of the master genome by increasing mutation rate (indicated with a thick black line in Fig. 3a). Although both models with and without complementation are structurally different, we will use the same values of the replication rate studied in Fig. 3a. As expected, the results show that the critical mutation rate without complementation is higher. From expression (9), using $f_{0}=0.9$ and $f_{1}=0.1$, we obtain $\mu_{c}=0.888 \ldots$ That is, considering complementation involves a lower mutation threshold because mutant genomes are taking advantage of the replicase translated from the master genomes and are, therefore, better competitors.

Finally, the equilibrium concentrations of the master and mutant genomes, computed numerically in the parameter space $(\mu, \gamma)$ using Eqs. (1-3), were in complete agreement with the previous results. We showed a decrease in the concentration of the master genomes at increasing mutation rate or decreasing $\gamma$, which is followed by an increase in the equilibria for the mutant genomes under such parameter changes (see Fig. 4).

In conclusion, we have shown that when complementation is incorporated into the picture, the critical mutation rate becomes independent of the replication rate of master and mutant genomes and only depends on the affinity of replicases for the master genome. From our results is 
also possible to conclude that complementation reduces the value of mutation rate at which transition to error catastrophe occurs.

\section{QUASISPECIES MODEL WITH DIPS}

Next, we focus our attention to a particularly interesting case in virology, namely the generation and amplification of defective interfering particles (DIPs) lacking the replicase gene. DIPs can only replicate in presence of a master genome that contributes with the wild-type replicase. This new model thus considers that the mutant genomes synthesized from the replication of the wild-type sequence produce a stronger interference because they are not producing mutant replicase which could contribute for replication of both genomes. As commented in the Introduction, experimental results suggest two different kinetic effects for DIPs: (i) the DIP, owed to its shorter genome length, replicates faster than the full-length master sequence and (ii) the DIP competes more successfully for interaction with the viral replicase machinery. Our model allows for the study of both effects: to simulate (i) we will set $r_{1}>r_{0}$, and by setting $\gamma<1 / 2$, we will simulate (ii).

The DIPs model follows a similar notation than the one presented and analyzed in Section II. It is worth mentioning that, for this system with DIPs, the term master sequence should be redefined as the one coding for the replicase, even though this replicase synthesizes faster or has stronger affinity for the mutant genomes. The model is given by the following set of three nonlinear ODEs:

$$
\dot{x}_{0}=(1-\mu) \gamma r_{0} x_{0} p_{0}-x_{0} \Phi(\mathbf{x}),
$$

$$
\dot{x}_{1}=p_{0}\left(\mu \gamma r_{0} x_{0}+(1-\gamma) r_{1} x_{1}\right)-x_{1} \Phi(\mathbf{x}),
$$

$$
\dot{p}_{0}=k_{0} x_{0}-p_{0} \Xi\left(p_{0}\right) \text {. }
$$

Here we also use the CP constraint, with $\sum_{i=0}^{1} x_{i}=1$ and $0 \leq p_{0} \leq 1$. The outflux terms are now given by

$$
\Phi(\mathbf{x})=p_{0}\left(\gamma r_{0} x_{0}+(1-\gamma) r_{1} x_{1}\right),
$$

and $\Xi\left(p_{0}\right)=k_{0} x_{0}$. This model is close to the one analyzed by Szathmáry (see section 2.3 in [28]). This author analyzed the dynamics of standard and defective interfering viruses with a structured deme model. Although in such a work a model with mutation was also presented, the characterization of the critical parameters and the bifurcation involved in the error-threshold were not thoughtfully studied. As a difference, our model considers in an explicit way the product of the template (i.e., replicase) and differential probabilities of interaction between the DIP and the wild-type replicase.
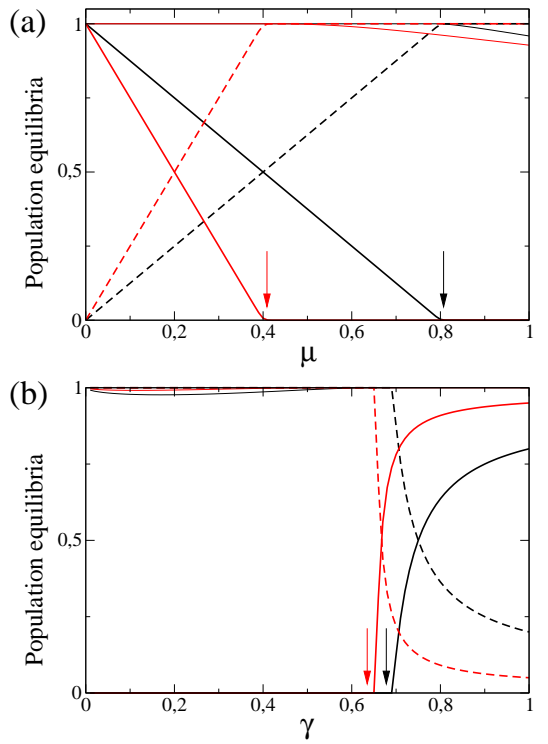

FIG. 6: Numerical values of the equilibria obtained from Eqs. (10)-(12) using $\mu$ and $\gamma$ as control parameters. The equilibrium concentration for the master and mutant genomes are indicated with solid and dashed thick lines, respectively. The solid thin lines correspond to the population equilibria of the replicase. In (a) we use $\gamma=0.75$ (red) and $\gamma=0.9$ (black). In (b) we use $\mu=0.05$ (red) and $\mu=0.2$ (black). In both plots $r_{0}=0.5, r_{1}=0.9, k_{0}=0.5$ and initial conditions $x_{0}(0)=1$ and $x_{1}(0)=p_{0}(0)=0$. The arrows indicate the values of $\mu_{c}$ (a) and $\gamma_{c}$ (b) for each analysis.

Let us analyze our model also reducing the system to a two-dimensional model using the linear relation obtained from the CP constraint, $x_{1}=1-x_{0}$. This system, also defined in the phase plane $\Gamma$ (see Section II), is given by

$$
\dot{x}_{0}=x_{0} p_{0} \Psi\left(x_{0}\right),
$$

$$
\dot{p}_{0}=k_{0} x_{0}\left(1-p_{0}\right) \text {. }
$$

with

$$
\Psi\left(x_{0}\right)=\left[(1-\mu) \gamma r_{0}-\left(\gamma r_{0} x_{0}+(1-\gamma)\left(1-x_{0}\right) r_{1}\right)\right] .
$$

The points in the straight line $\left\{\left(0, p_{0}\right)\right\}$ are fixed points. For this case, the asymptotic state of the master sequence is the extinction. Hence, it can be interpreted as a ("onedimensional") absorbing state tied to the error catastrophe. This line of equilibria involves the extinction of the master genomes with the presence of wild-type replicase. This state is found because during the transient dynamics towards the extinction of the master genome, wild-type replicase molecules are still produced. As the wild-type replicases are not degraded, they remain in the system and, asymptotically, they reach a non-trivial value, although the master genomes become extincted. If $\left(x_{0}, p_{0}\right)$ 

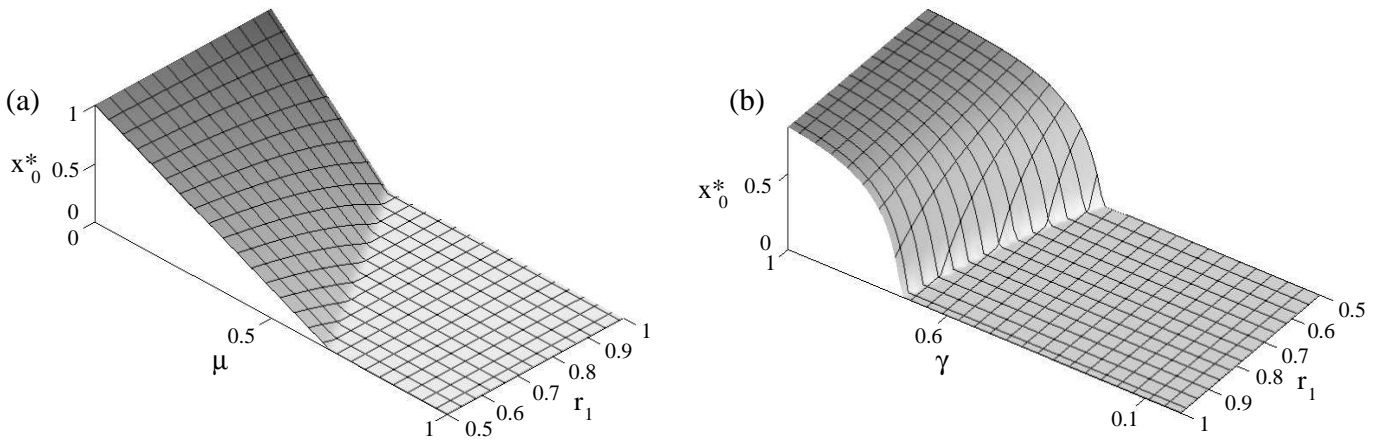

FIG. 7: Equilibrium concentration of the master genomes obtained numerically from Eqs. (10)-(12) in the parameter spaces $\left(\mu, r_{1}\right)(\mathrm{a})$, and $\left(\gamma, r_{1}\right)(\mathrm{b})$. In (a) we use $\gamma=0.75$ and in (b) $\mu=0.2$. In both plots we set $k_{0}=0.5$ and $r_{0}=0.5$. Initial conditions were: $x_{0}(0)=1$ and $x_{1}(0)=p_{0}(0)=0$.

is a fixed point with $x_{0} \neq 0$, from Eq. (14) $p_{0}=1$, and then from Eq. (13) we obtain

$$
x_{0}^{*}=\frac{(1-\mu) \gamma r_{0}-(1-\gamma) r_{1}}{\gamma r_{0}-(1-\gamma) r_{1}} .
$$

Then, assuming that $0<x_{0}^{*} \leq 1$ we have another fixed point:

$$
\left(x_{0}^{*}, 1\right) \equiv\left(\frac{(1-\mu) \gamma r_{0}-(1-\gamma) r_{1}}{\gamma r_{0}-(1-\gamma) r_{1}}, 1\right)
$$

The Jacobian matrix for this model is

$$
J=\left(\begin{array}{cc}
p_{0}\left(\Psi\left(x_{0}\right)+x_{0} \Psi^{\prime}\left(x_{0}\right)\right) & x_{0} \Psi\left(x_{0}\right) \\
k_{0}\left(1-p_{0}\right) & -k_{0} x_{0}
\end{array}\right) .
$$

At the point $(0,0)$ both eigenvalues are zero (i.e., degenerate case). The linearized equation is unstable,

$$
\left(\begin{array}{l}
\dot{u} \\
\dot{v}
\end{array}\right)=\left(\begin{array}{cc}
0 & 0 \\
k_{0} & 0
\end{array}\right)\left(\begin{array}{l}
u \\
v
\end{array}\right)
$$

$\dot{u}=0$ and $\dot{v}=k_{0} u$, and has solution $u(t)=C_{1}$ and $v(t)=k_{0} C_{1} t+C_{2}$. These solutions with $u(0)=C_{1} \neq 0$ go away from any neighborhood of $(0,0)$. At the points $\left(0, p_{0}\right)$,

$$
J=\left(\begin{array}{cc}
p_{0}(\Psi(0)) & 0 \\
k_{0}\left(1-p_{0}\right) & 0
\end{array}\right),
$$

and the two eigenvalues are $\lambda_{1}=0$ and $\lambda_{2}=p_{0}[(1-$ u) $\left.\gamma r_{0}-(1-\gamma) r_{1}\right]$. Note that the points $\left(0, p_{0}\right)$ will be the ones involved in the extinction of the master sequence for the model with DIPs. If

$$
(1-\mu) \gamma r_{0}-(1-\gamma) r_{1}<0,
$$

these points have a one-dimensional invariant stable manifold. From the previous inequation we can obtain the critical values responsible of the error threshold, which are given by

$$
\mu_{c}=1-\left((1-\gamma) r_{1} / \gamma r_{0}\right)
$$

and

$$
\gamma_{c}=r_{1} /\left(r_{0}(1-\mu)+r_{1}\right)
$$

Then, if $\mu>\mu_{c}$ or $\gamma<\gamma_{c}$ the master genomes will disappear of the population, which will be completely dominated by the mutant DIPs. The previous critical values are in complete agreement with numerical simulations. In Fig. 5(a) we repeated the phase diagram shown in Fig. 2(a), where we represented (in gray) the pairs of the parameters $(\gamma, \mu)$ involving the extinction of the master genomes. Note that the critical boundary displayed with the solid black line perfectly fits with the values of $\gamma_{c}$ and $\mu_{c}$ previously characterized. For the other fixed point $\left(x_{0}^{*}, 1\right), \Psi\left(x_{0}^{*}\right)=0$, and

$$
J=\left(\begin{array}{cc}
x_{0}^{*} \Psi^{\prime}\left(x_{0}^{*}\right) & 0 \\
0 & -k_{0} x_{0}^{*}
\end{array}\right) .
$$

The eigenvalues for this case are $\lambda_{1}=-k x_{0}^{*}<0$ and $\lambda_{2}=x_{0}^{*} \Psi^{\prime}\left(x_{0}^{*}\right)$, with

$$
x_{0}^{*} \Psi^{\prime}\left(x_{0}^{*}\right)=-(1-\mu) \gamma r_{0}+(1-\gamma) r_{1} .
$$

With this basic information obtained only from the linear study we can see that

$$
(1-\mu) \gamma r_{0}-(1-\gamma) r_{1}
$$

plays an important role in the dynamics of the system. If expression (16) is negative, the points of the straight line $\left\{\left(0, p_{0}\right)\right\}$ are (weakly) attractive. However, with a positive value for expression $(16)$ the point $\left(x_{0}^{*}, 1\right)$ is attractive. The previous analytical results have been checked numerically. As previously discussed, the straight line $\left\{\left(0, p_{0}\right)\right\}$ contains fixed points, as it is shown in Fig. 5(b) in the scenario where $\left\{\left(0, p_{0}\right)\right\}$ has a stable invariant manifold (i.e., $\left.(1-\mu) \gamma r_{0}-(1-\gamma) r_{1}<0\right)$. Note that different 
initial conditions achieve different asymptotic values in such a straight line, in which the master genomes become extinct. The other possible scenario, in which the master genomes survive and coexist with the DIPs, is shown in Figs. 5(c) and 5(d). For these two cases $\mu<\mu_{c}$ and $\gamma>\gamma_{c}$. The crossing of these critical values also involves transcritical-like bifurcations. This type of bifurcations, also found for the general model analyzed in Section II, is displayed in Fig. 6.

The increase of mutation rate in the range $\mu<\mu_{c}$ involves a linear decrease in the concentration of the master genome and a linear increase of the mutant genomes concentration (Fig. 6(a)). Moreover, the decrease of $\gamma$ involves a decrease in the equilibrium concentration of the master genomes, and when $\gamma<\gamma_{c}$ the population of master genomes is not able to survive at all (Fig. 6(b)). The previous results suggest that under the condition that the DIP has a higher affinity in the binding with the replicase, even for very small mutation rates (e.g., with $\mu=0.05$ as shown in Fig. 6(b) in red), these mutant DIPs will outcompete the master ones. Actually, the phase diagram of Fig. 5(a) shows that below $\gamma<0.5$, the mutant DIPs outcompete the master one for any mutation rate (a similar result was also found in Section II).

We finally analyzed the effect of increasing the replication rate of the mutant genomes in the range $r_{0}<r_{1} \leq 1$. The increasing of $r_{1}$ might correspond to the study of several DIPs with shorter genome lengths, assuming a linear relation between genome length and replicative speed for mutant genomes. These analyses were performed by computing numerically the equilibrium values of the master genomes in the parameter spaces $\left(\mu, r_{1}\right)$ (Fig. $\left.7(\mathrm{a})\right)$ and $\left(\gamma, r_{1}\right)$ (Fig. $\left.7(\mathrm{~b})\right)$. In both graphs it can be seen that the master genomes become extinct as the values of $\mu_{c}$ and $\gamma_{c}$ are crossed. Moreover, as expected, we showed that an increase of the replication speed for the mutant DIPs involved an earlier extinction in both parameter spaces. That is, the values of $\mu_{c}$ decreases as $r_{1}$ grows, and the values of $\gamma_{c}$ increase at increasing $r_{1}$.

In conclusion, the previous results show that when DIPs are considered as a particular case of mutants, the critical mutation rate depends both on the replication rates and binding affinities. Furthermore, the more replicative advantage of the DIPs relative to the master genomes, the lower the critical mutation rate.

\section{DISCUSSION}

The quasispecies theory [8-10] has become the paradigmatic theoretical framework for the study of the dynamics and evolution of error-prone populations of replicators like RNA viruses. In the light of this theory, several interesting results have been reported, among them, the existence of the so-called error-threshold $[8,11,15]$. The error threshold has been defined as the value of the mutation rate below which populations equilibrate in a traditional mutation-selection balance and above which the population experiences an error catastrophe through excessive deleterious mutations [11]. The standard quasispecies model directly associates the fitness of a given replicator to its genotype. Translating this assumption to real viral populations may be cumbersome, since it has been amply demonstrated that the phenotype of a viral particle does not necessarily reflects the genotype it contains [17-20].

Complementation between different viral genomes by means of trans interactions between different virus genotypes has been shown to be of importance in the dynamics of viral replication and cell infection, as shown in some theoretical studies [27-29]. Previous modeling attempts of viral complementation, including interaction between DIPs and full viral genomes, focused in the ecological interactions [25-27] or population genetics [24] of the different genotypes and the host cell. The main difference between these previous analyses and this work, is that here we studied two simple quasispecies models considering complementation during the replication of master (wild-type) and mutant genomes and the effect of such trans interactions on the error-threshold.

We first analyzed a model with master and mutant genomes coding for wild-type and mutant (functionally impaired) replicases. Due to complementation, both genomes can be replicated by both replicases, with differential interactions probabilities between master and mutant genomes with the whole pool of replicases (parametrized with $\gamma$ ) synthesized from both genome classes. Then, we analyzed a different model considering a particular case of complementation in which DIPs (see the review [32]) replicate faster due to their shortened genomes. Moreover, we can also incorporate into this model differential binding affinities for the unique, wild-type replicase.

For both models we characterized the coexistence scenario between master and mutant genomes, as well as the critical values of mutation and $\gamma$ involving the entry into error catastrophe. That is, the values of such parameters with the full population dominated by the mutant genotypes. We found that the error-catastrophe scenario in the parameter space $(\mu, \gamma)$ was enlarged in the complementation model compared with the standard quasispecies model. Furthermore, we also found that the parameter space in which error-catastrophe occurs was even larger for the DIPs model than for the simple complementation one. We have also characterized the errorthreshold transition when crossing $\mu=\mu_{c}$ and $\gamma=\gamma_{c}$ as transcritical-like bifurcations in both systems under study, suggesting the presence of continuous phase transitions in the qualitative shift from viable master genomes to error catastrophe. Such a phase transition was characterized in previous works for the standard quasispecies model [39]. However, other works with quasispecies models defined on sharp, single-peak landscapes have shown the presence of first-order phase transitions [43] as well as the possibility of both transitions occurring in the same system at increasing mutation rates [44]. As mentioned 
in the Introduction, our system might be equivalent to a hypercycle system with replicase-mediated replication. The consideration of replicases, as we have shown, might involve the presence of a smoothed phase transition, which is in contrast with the governing bifurcations of ribozyme-like hypercycles, in which the extinction of the replicons is governed by saddle-node bifurcations [45, 47] and by absorbing first-order phase transitions [46].

Focusing in our first studied model, we have compared the critical mutation rate under complementation with the critical mutation obtained from a simple standard quasispecies model, also under the Swetina-Schuster fitness landscape. We have shown that the error threshold occurs at a lower mutation rate with complementation. We have also determined an error-threshold mediated by the binding probability of genomes with the replicases. Our results suggest that even for viruses that may be replicating far away from the mutation threshold (i.e., $\mu<<\mu_{c}$ ), the population can be forced to error catastrophe by the existence of mutant RNA genomes for which the replicase may show an increased affinity.

For mathematical convenience, we have used the Swetina-Schuster single peak fitness landscape [42] in our models, despite its clear lack of biological realism. In a previous study, we have shown that different fitness landscapes, with variable degrees of epistasis, have substantially different effects on the error threshold [48]. Therefore, a logical follow up of the present study would be to explore the combined effect of epistasis and complementation among mutations on the properties of the error threshold. Gao and Feldman [24] have recently developed a population genetic model of the effect of trans complementation with epistasis in viral dynamics. These authors found that the election of the epistasis function (i.e., synergistic or antagonistic) have profound effects on the genetic composition of complementing viral genomes. For instance, synergistic epistasis tends to strengthen the selection against the combinations of less-fit mutations, while frequent coinfection generally weakens the selection against all mutations, with the result that the master and single mutants constitute a major proportion of the virus population, and the stronger the complementation is, the lower the frequency of master genomes.

By contrast, antagonistic epistasis among deleterious mutations produce a much more complex picture and depends on whether antagonistic epistasis takes the form of magnitude or sign epistases [49]. With sign antagonistic epistasis (e.g., if both the master and high-order mutants are fitter than the single mutants), master genomes tend to disappear and the population is dominated by genotypes with large numbers of mutations. However, with magnitude antagonistic epistasis (e.g., the strength of epistasis depends on the genetic background but the sign is unconditional), the master, single and double mutants dominate the population. These analytical results suggest that with complementation the error threshold may occur at lower critical mutation rates with sign antagonistic epistasis than with magnitude antagonistic epistasis and than with synergistic epistasis.

\section{Acknowledgments}

The authors want to thank the useful comments and suggestions of Ernest Fontich. This work has been funded by the Human Frontier Science Program Organization grant RGP12/2008 and by the Spanish Ministerio de Ciencia e Innovación grant BFU2009-06993. We also acknowledge support from the Santa Fe Institute.
[1] Nowak, M. A. (1992) What is a quasispecies? Trends Ecol. Evol. 7, 118-21

[2] Domingo, E. and Holland, J. J. (1997) RNA virus mutations and fitness for survival. Annu. Rev. Microbiol. 51, 151-78

[3] Domingo, E., Biebricher, C. K., Eigen, M. and Holland, J. J. (2001) Quasispecies and RNA virus evolution: Principles and Consequences, Georgetown, TX: Landes Bioscience

[4] Domingo, E., Holland, J. and Ahlquist, P. (1988) RNA genetics (CRC Press, Boca Raton, Fla)

[5] Domingo, E., Holland, J. (1994) Mutations and rapid evolution of RNA viruses (ed. S., M. S.)(Raven Press, New York)

[6] Domingo, E. (2000) Viruses at the edge of adaptation. Virology 270, 251

[7] Domingo, E., Sabo, D., Taniguchi, T., Weissmann, C. (1978) Nucleotide sequence heterogeneity of an RNA phage population. Cell 13, 735-44

[8] Eigen, M. (1971) Self-organization of matter and the evolution of biological macromolecules. Naturwissenschaften

\section{$\mathbf{5 8}, 465-523$}

[9] Eigen, M., McCaskill, J. and Schuster, P. (1988) Molecular quasi-species. J. Phys. Chem. 92, 6881-6891

[10] Eigen, M., McCaskill, J. and Schuster, P. (1989) The Molecular Quasispecies. Adv. Chem. Phys. 75, 149-263

[11] Bull, J. J., Meyers, L. A., and Lachman, M. (2005) Quasispecies Made Simple. PLoS Comp. Biol. 1:6, 0450-0460

[12] Eigen, M. (2003) Viral quasispecies. Sci. Am. 269, 42-49

[13] Biebricher, C. K. and Eigen, M. (2005) The errorthreshold. Virus Res. 107, 117-27

[14] Elena, S.F. and Sanjuán, R. (2007) Virus evolution: insights from an experimental approach. Annu. Rev. Ecol. Evol. Syst. 38: 27-52

[15] Schuster, P. (1994) How do RNA molecules and viruses explore their worlds? In: Cowan, G. A., Pines, D., Meltzer, D. (Eds.), Complexity: Metaphors, Models and Reality. Addison-Wesley, Reading, MA, p. 384-418

[16] Wilke, C. O. and Novella, I. Phenotypic mixing and hiding may contribute to memory in viral quasispecies. BMC Microbiol. 3:11

[17] Novick, A. and Szilard, L. (1951) Virus strains of identi- 
cal phenotype but different genotype. Science 113, 34-35

[18] Brenner, S. (1957) Genetic control and phenotypic mixing of the adsorption cofactor requirement in bacteriophages T2 and T4. Virology 3, 560-574

[19] Huang, A. S., Palma, E. L., Hewlett, N. and Roizman, B. (1974) Pseudotype formation between enveloped RNA and DNA viruses. Nature 252, 743-45

[20] Holland, J. J., de la Torre, J. C., Steinhauer, D. A., Clarke, D., Duarte, E. and Domingo, E. Virus mutation frequencies can be greatly underestimated by monoclonal antibody neutralization of virions. J Virol 63, 5030-5036

[21] Cicin-Sain, L., Podlech, J., Messerle, M., Reddehase, M.J., Koszinowski, U.H. (2005) Frequent coinfection of cells explains functional in vivo complementation between cytomegalovirus variants in the multiply infected hosts. J. Virol. 79: 9492-9502

[22] García-Arriaza, J., Manrubia, S.C., Toja, M., Domingo, E., Escarmís, C. (2004) Evolutionary transition towards defective RNAs that are infectious by complementation. J. Virol. 78: 11678-11685

[23] Fraile, A., Sacristán, S., García-Arenal, F. (2008) A quantitative analysis of complementation of deleterious mutants in plant virus populations. Span. J. Agricult. Res. 6: 195-200

[24] Gao, H., Feldman, M.W. (2009) Complementation and epistasis in viral coinfection dynamics. Genetics 182: 251-263

[25] Bangham, C.R.M. and Kirkwood, T.B.L. (1990) Defective interfering particles: effects in modulating virus growth and persistence. Virology 179: $821-826$

[26] Frank, S.A. (2000) Within-host spatial dynamics of viruses and defective interfering partiles. J. Theor. Biol. 206: $279-290$

[27] Kirkwood, T. B. L. and Bangham, C. R. M. (1994) Cycles, chaos, and evolution in virus cultures: A model of defective interfering particles. Proc. Natl. Acad. Sci. USA 91, 8685-89

[28] Szathmáry, E. (1992). Natural Selection and Dynamical Coexistence of Defective and Complementing Virus Segments. J. Theor. Biol. 157: 383-406

[29] Szathmáry, E. (1993). Co-operation and Defection: Playing the Field in Virus Dynamics. J. Theor. Biol. 165: 341-356

[30] Holland, J.J., Villareal, L.P., Breindl, M. (1976) Factors involved in the generation and replication of rhabdovirus defective T particles. J. Virol. 17: 805-815

[31] Damayanti, T.A., Nagano, H., Mise, K., Furusawa, I., Okuno, T. (1999) Brome mosaic virus defective RNAs generated during infection of barley plants. J. Gen. Virol. 80: $2511-2518$

[32] Marriott, A. C., and Dimmock, N. J. (2010) Defective interfering viruses and their potential as antiviral agents. Rev. Med. Virol. 20: 51-62

[33] Horodyski, F.M., Nichol, S.T., Spindler, K.R., Holland, J.J. (1983) Properties of DI particles resistant mutants of vesicular stomatitis virus isolated from persistent infections and from undiluted passages. Cell 33: 801-810
[34] DePolo, N.J., Holland, J.J. (1986) Very rapid generation/amplification of defective interfering particles by vesicular stomatitis virus variants isolated from persistent infections. J. Gen. Virol. 67: 1195-1198

[35] DePolo, N.J., Giachetti, C., Holland, J.J. (1987) Continuing coevolution of virus and defective interfering particles and of viral genome sequences during undiluted passages: virus mutants exhibiting nearly complete resistance to formerly dominant defective interfering particles. J. Virol. 61: 454-464

[36] Pattnaik, A.K., Wertz, G.W. (1991) Cells that express all five proteins of vesicular stomatitis virus from cloned cDNAs support replication, assembly, and budding of defective interfering particles. Proc. Natl. Acad. Sci. USA 88: $1379-1383$

[37] Jacobson, S. and Dutko, F. J. and Pfau, C. J. (1979) Determinants of spontaneous recovery and persistence in MDCK cells infected with lymphocytic choriomeningitis virus. J. Gen. Virol. 44, 113-121

[38] Eigen, M., Schuster, P. (1979) The Hypercycle. A Principle of natural Self-organization. Springer-Verlag

[39] Tarazona, P. (1992) Error thresholds for molecular quasispecies as phase transitions: From simple landscapes to spin-glass models. Phys Rev A 45:8, 6038-6049

[40] Campos, P.R.A., J.F. Fontanari and P.F. Stadler (2000) Error propagation in the hypercycle, Phys. Rev. E 61: 2996-3002

[41] Silvestre, A. M. M., and Fontanari, J. F. (2008) The information capacity of hypercycles. J Theor Biol 254, 804-806

[42] Swetina, J. and Schuster, P. (1982). Self-replication with errors. A model for polynucleotide replication. Biophys. Chem. 16: 329

[43] Pastor-Satorras, R. and Solé, R. V. (2001). Field theory for a reaction-diffusion model of quasispecies dynamics. Phys. Rev. E 64: 051909

[44] Saakian, D. B., Biebricher, C. K. and Hu, C-K. (2009) Phase diagram for the Eigen quasispecies theory with a truncated fitness landscape. Phys. Rev. E 79: 041905

[45] Sardanyés, J. (2008) Error threshld ghosts in a simple hypercycle with error prone self-replication. Chaos, Solitons \& Fractals, 35, 313-319

[46] Sardanyés, J. and Solé, R. V. (2006) Bifurcations and phase transitions in spatially-extended two-member hypercycles. J Theor Biol 243, 468-482

[47] J.C. Nuño, F. Montero and F.J. de la Rubias. (1993) Influence of external fluctuations on a hypercycle formed by two kinetically indistinguishable species, J theor Biol 165, pp. 553575

[48] Sardanyés, J., Solé, R. V. and Elena, S. F. Replication Mode and Landscape Topology Differentially Affect RNA Virus Mutational Load and Robustness. J. Virol. 83(23): 12579-89

[49] Weinreich, D., Watson, R., Chao, L. (2005) Sign epistasis and genetic constraint on evolutionary trajectories. Evolution 59: 1165-1174 